\title{
Neck and Shoulders Motion Range after Neck Dissection
}

\author{
Ljiljana Širić $^{1 *}$, Vesna Širić ${ }^{2}$, Marinela Rosso ${ }^{3}$, Željko Širić $^{4}$, Željko Kotromanovići $^{1}$ and Hrvoje Mihalj ${ }^{1}$ \\ ${ }^{1}$ Department of Otorhinolaryngology and Head and Neck Surgery, University Hospital Centre Osijek, Osijek, Croatia \\ ${ }^{2}$ Chair of Physical Training and Health Culture, Faculty of Law, University of J.J. Strossmayer Osijek, Osijek, Croatia
}

${ }^{3}$ Polyclinic Rosso, Osijek, Croatia

${ }^{4}$ Chair of Social Sciences and Humanities, Faculty of Electrical Engineering, Computer Science and Information Technology University of J.J. Strossmayer Osijek, Osijek, Croatia

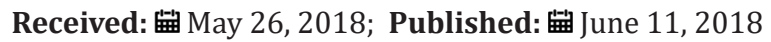

*Corresponding author: Ljiljana Širić, Department of Otorhinolaryngology and Head and Neck Surgery, University Hospital Centre Osijek, Croatia

\begin{abstract}
Neck dissection is a complex surgical procedure which leaves multiple consequences on the person to whom it is made. The aim was to measure the degree of damage to neck and shoulders motor function in patients who underwent neck dissection.

Materials and methods: 30 subjects divided into two groups depending on the dissection made (16 (53.3\%) of selective and 14 (46.6\% radical) accounted for both sexes (3 (10\% of women and 27 (90\%) of men) in average age of 64.2 years who underwent neck dissection. Digital goniometer was used to measure subject's maximum range of motion on 7 variables ( 4 variables for the neck movements and 3 variables for the shoulder movements), of which 5 were bilateral. The results are ranked with in the category of a certain degree of damage depending on the standardized values of the maximum amplitude at the non-operated population with normal function of motion.
\end{abstract}

Results: Significant difference was found in the values of the amplitude of shoulder flexion, extension and abduction between groups at a significance level of 5\% and in the confidence interval of 95\%.

Conclusion: Rehabilitation is necessary after neck dissection and kinesiotherapy can be an important rehabilitation method for patients.

Keywords: Neck Dissection; Neck Motion Amplitude; Range of Motion; Rehabilitation; Shoulder Motion Amplitude.

Abbreviations: SND: Selective Neck Dissection; RND: Radical Neck Dissection; NF: Neck Flexion; NE: Neck Extension; NR: Neck Rotation; NLF: Neck Lateral Flexion; SF: Shoulder Flexion; SE: Shoulder Extension; SA: Shoulder Abduction; N: Number of Samples; x: Arithmetic Mean; SD: Standard Deviation; t: T value; df: Degree Of Freedom; F: F value; p: P value.

\section{Introduction}

Dissection is a very complex surgical procedure on the neck where the most of vital structures of the front and lateral sides of the neck are and is carried out independently or with a basic procedure in patients with malignant head and neck tumors. It is done to treat regional metastases on neck lymph nodes. There are several types of dissection, depending on the surgical approach. Selective neck dissection includes one or more regions of the lymph nodes, depending on the localization of primary tumors. Radical dissection includes the lymph nodes within all five neck regions, removal of internal jugular vein, sternocleidomastoid muscle, and accessory nerve. Modified radical dissection involves removing lymph nodes of all five neck regions with exemption of one or more non lymphatic structures [1]. Preserving the nerve does not necessarily imply its normal function. Dissection leads to loss of motor strength, muscle volume and sensation. Some of the consequences are painful and lowered shoulder, neck, shoulder or upper chest insensitivity, neck pain, decreased neck and shoulders mobility, inability or difficulty in raising hands above the head, reduced arm strength, and Horner's syndrome [2-4]. 
Such functional impairment is not uncommon, and out of the total number, $5 \%$ of damage occurs by chance [5]. Dysfunction comes to the fore in the later postoperative course which significantly determines the physical and social functioning, reduces working capacity and affects the quality of life [6]. Primary rehabilitation is carried out directly after the surgical procedure in a hospital, the secondary should be carried out after the release in order to further improve and tertiary in order to maintain the achieved condition. These patients have rarely been sent to a specialized facility after hospitalization so few exercise by themselves at home, and most do not exercise at all [6]. Normal values of the amplitude of neck flexion range from $0-100^{\circ}$, extensions from 0 to $25^{\circ}$, lateral flexions to the left and right sides from 0 to $40^{\circ}$, and neck rotation to both sides from $40^{\circ}$ to $45^{\circ}$. Normal values of the amplitude of shoulder flexion are $100^{\circ}$ and more, the shoulder extensions $60^{\circ}$ or more, and shoulder abduction from $160^{\circ}$ to $180^{\circ}$ [7]. Kinesiotherapy, which uses movement to mobilize existing physiological mechanisms and function, can be an important method for the rehabilitation of certain pathological conditions arising as a result of a surgical procedure. Exercising under the expert guidance and supervision of a kinesiotherapists, allows patients the establishment of optimal motor functions according to the anatomical and functional damage and thus easier return to the life or work environment. According to the current available data, within an interdisciplinary team for treating head and neck cancers, as well as for the rehabilitation of the consequences of surgical treatment of diseases, a specialist of this profile is not detected.

\section{Materials and Methods}

\section{Study design}

This is a cross-sectional study which included the initial measurement of the movement range of neck and shoulder after neck dissection and before rehabilitation.

\section{Ethical procedures}

The procedures were performed according to the Declaration of Helsinki and approved by the Ethics Committee of the University Hospital Center Osijek on January 23th, 2017, under the protocol number R2-10203/2017. The participants signed informed consent to participate in the research.

\section{Setting}

The study was conducted at the Department of Otorhinolaryngology and Head and Neck Surgery in University Hospital Centre Osijek during 2017 on patients with head and neck tumors.

\section{Participants}

A sample of 30 patients consisted of both sexes, 3 (10\%) women and 27 (90\%) men, average age of 64.2 years. The study included people who underwent neck dissection for malignant diseases of the throat, and excluded people who preoperatively had reduced mobility and / or soreness of neck and shoulder of different etiology. Subjects were divided into two groups depending on the type of dissection: the first group consisted of 16 (53.3\%) subjects who underwent selective dissection, and the other $14(46.6 \%)$ subjects who underwent radical dissection.

\section{Measurement and variables}

Digital goniometer (Warenhandel GmbH \& Co., Germany) was used to measure subject's maximum amplitude of neck and shoulders motions. Total of seven variables was measured: 4 variables for the neck movements - flexion, extension and rotation and lateral flexion bilaterally and 3 variables for shoulder movements - flexion, extension and abduction bilaterally. The obtained data were compared to standardized values of maximum amplitude in individuals with normal function, and between groups. For ease of processing, depending on the level of the maximum amplitude, the results were scored and ranked in five groups where 1 means complete function reduction, 2 heavy reduction, 3 moderate reductions, 4 light reduction, and 5 normal function. The categorization of each individual movement was done in a way that the maximum value of the amplitude was divided by five to obtain the same class limits.

\section{Statistical methods}

Data were statistically analyzed using Statistical Package for the Social Science for Windows (SPSS Inc. 16.0, Chicago, IL, SAD). A descriptive analysis of the data was performed, resulting in frequencies and percentages. From nominal and ordinal variables the analysis of variance for independent samples was performed, and the normality of distribution was tested with KolmogorovSmirnov test.

\section{Results}

On variables of neck flexion 10 subjects have a preserved amplitude of movement (33.3\%), 20 have a light dysfunction (66.6\%), and moderate/severe dysfunction or complete loss of function is not recorded in any subjects. On the variables of neck extension 4 (13.3\%) subjects have a proper amplitude of movement, $17(56.6 \%)$ have a light reduction in amplitude, 7 (23.3\%) have moderate, and $2(6.6 \%)$ severe reduction in amplitude. Also, no patients with complete inability of movement were recorded. On the variable of neck rotation to both sides most subjects, 10 of them (33.3\%), have a moderate dysfunction of movement, 9 (30\%) have severe, and 7 (23.3\%) light dysfunction (Table 1).

No patients with absolute impossibility of neck rotation were recorded, and 4 of them (13.3\%) have a preserved movement function. In lateral neck flexion proper amplitude have 7 (23.3\%) of subjects, and 2 of them (6.6\%) have a complete loss. $9(30 \%)$ subjects, most of them, have moderate deviation, 8 (26.6\%) have light deviation, and 4 (13.3\%) have a severe deviation. On variables of shoulder flexion most subjects have a light dysfunction, 14 of them $(46.6 \%)$, followed by $11(36.6 \%)$ with moderate dysfunction and $2(6.6 \%)$ subjects with severe dysfunction. $3(10 \%)$ subjects have a proper shoulder flexion movement, and none of the subjects has a complete loss of function. Regarding the shoulder extension, 
proper movement, loss of function and severe dysfunction have 3 $(10 \%)$ of subjects. Most of them have a moderate dysfunction, 11 of them $(36.6 \%)$, followed by $10(33.3 \%)$ with severe dysfunction. None of the subjects has a preserved function of shoulder abduction, as well as light dysfunction. $3(10 \%)$ subjects have a complete loss of function, 12 (40\%) have a severe dysfunction, and $15(50 \%)$ a moderate dysfunction. All subjects had a constant feeling of pain in the neck and a significant pain in shoulder, feeling of tightness while initiating movement, and 7 (23.3\%) of them, who underwent radical dissection, had a significantly lowered shoulder and spinal pain (Table 1). Analysis of variance, on the variables of neck movement in all directions (flexion, extension, rotation and lateral flexion) showed no statistically significant difference between subjects with selective or radical neck dissection, while on the variables of shoulder movement (bilateral flexion, extension and abduction) a statistically significant difference between groups was determined, at a significance level of $5 \%$ in the confidence interval of $95 \%$.

Table 1: Significance of the difference in the amplitude of neck and shoulders motions between selective and radical neck dissection.

\begin{tabular}{|c|c|c|c|c|c|c|c|c|c|}
\hline \multirow[b]{2}{*}{ variable } & \multirow[b]{2}{*}{$\mathbf{N}$} & \multicolumn{2}{|c|}{ SND } & \multicolumn{2}{|c|}{ RND } & \multirow{2}{*}{$\mathbf{t}$} & \multirow{2}{*}{ df } & \multirow{2}{*}{$\mathbf{F}$} & \multirow{2}{*}{$\mathbf{p}$} \\
\hline & & $\mathbf{x}$ & SD & $\mathbf{x}$ & SD & & & & \\
\hline NF & 30 & 4,312 & 4787 & 4,357 & 4972 & -250 & 28 & 0,244 & 0,804 \\
\hline $\mathrm{NE}$ & 30 & 3,750 & 6831 & 3,857 & 7703 & -404 & 28 & 0,095 & 0,689 \\
\hline NR & 60 & 3,343 & 9708 & 3,214 & 11,338 & 477 & 58 & 1,253 & 0,635 \\
\hline NLF & 60 & 3,400 & 11,960 & 3,202 & 11,762 & 1,577 & 58 & 3,841 & 0,120 \\
\hline SF & 60 & 3,633 & 7804 & 2,113 & 4801 & 1,945 & 58 & 4,896 & 0,050 \\
\hline SE & 60 & 2,850 & 10,865 & 1,653 & 325 & 2,160 & 58 & 3,111 & 0,035 \\
\hline SA & 60 & 25,667 & 6474 & 15,667 & 2532 & 2,441 & 58 & 0,652 & 0,01 \\
\hline
\end{tabular}

\section{Discussion}

The results indicate a very frequent extensive reduction of shoulder functions as confirmed by the results of previous studies $[8,9]$. The study which included 65 subjects showed that $77 \%$ of subjects had a shoulder dysfunction of different degree and $23 \%$ of subjects had a preserved shoulder function [10]. Milenović et al. [2] examined the loss of function of the brachial plexus on the basis of the presence of pain and weakness in the shoulder, arm or hand, and the possibility of shoulder abduction, and $14 \%$ of subjects had a preserved function of the respected nerve. The cause of this dysfunction is usually the manipulation of spinal nerves which secondarily results in an atrophy of trapeziums [11,12]. Partially there is always a preserved function of shoulder regardless of nerve damage, because the lower part of the trapeziums muscle is innervated by C2-C4 nerves. In a study by Spiro et al. [13] $13 \%$ of subjects with a resection of $n$. vagus after radical neck dissection were recorded, and bilateral paralysis of the diaphragm was recorded by Yaddanapudi et al. [14,15]. In 15\% of cases with preserved nerve dysfunction of movement occurs due to high sensitivity and small dimensions of the nerve during preparation. Milenović et al. [2] \& Jong et al. [12] during their research found no significant difference in motor nerves function depending on the type of dissection made, but they examined nerve function neurologically, without taking into account further motor function of the muscle innervated by the examined nerve $[10,11]$.

\section{Limitations of the Study}

The main limiting factor is a small sample. There is also a problem of additional homogenization of groups because each type of neck dissection has subsets.

\section{Conclusion}

This study showed the highest or significant reduction in amplitude in an attempt of shoulder abduction where the degree of damage varied from moderate and severe to complete. This is the only variable without subjects with completely normal function or light dysfunction. Also, on the same variable statistically most significant difference between subjects who underwent selective or radical dissection was found. The best preserved amplitude was measured during the neck flexion regardless of the type of dissection made, where a statistically significant difference between groups was not found. However, these results should be interpreted with caution due to the relatively small sample size, the complexity of neck dissection and a large number of dependent variables that have a potential impact on the measured variable. Daily continuous exercise can alleviate the consequences of this mutilating procedure and the results of exercise would certainly be better if the exercises for strengthening the neck muscles and shoulder area would be performed individually under the supervision of a kinesiotherapist. Back in the 1981 Tissot wrote that the movement can replace any medical therapeutic remedy while the remedies cannot replace movement.

\section{References}

1. Virag M. (2006) The life of a patient with a cancer of the head and neck Croatian society for maxillofacial, plastic and reconstructive treatment of head and neck tumors. How to improve the quality of life of patients with head and neck cancer. Croatian society for maxillofacial, plastic and reconstructive surgery of the head and neck 5-12.

2. Milenović A (2006) The function of brain nerves and nerves of the cervical plexus after the dissection of the neck. In: How to improve the quality of head and neck surgery 32-33. 
3. Dedivitis RA, Guimaraes AV, Pfuetzenreiter EG, Castro MA (2011) Neck dissection complications. Braz J Otorhinolaryngol 77(1): 65-69.

4. Lukšić I, Virag M, Aljinović Ratković N (2006) Physical therapy and rehabilitation after radical neck dissection. In: How to improve the quality of life of patients with head and neck cancer. Croatian Society for Maxillofacial, Plastic and Reconstructive Surgery of Head and Door 57-60.

5. Million RR, Cassisi NJ (1994) Management of head and neck cancer: a multidisciplinary approach. 2 izd Philadelphia: Well, Lippincott 75-142.

6. Orihovac Ž (2006) Assessment of working ability. In: How to improve the quality of life of patients with head and neck cancer. Croatian Society for Maxillofacial, Plastic and Reconstructive Surgery of Head and Door 61-62.

7. Stephens K (2017) Normal Neck Range of Motion.

8. Sobol S, Jensen C, Sawyer W (1985) Objective comparison of physical dissection after neck dissection. Am J Surg 150(4): 503-509.

9. Leipzig B, Suen JY, English JL, Barnes J, Hooper M (1983) Functional evaluation of spinal accessory nerve after neck dissection. Am J Surg 146(4): 526-530
10. Carew JF, Spiro RH (1997) Extended neck dissection. Am J Surg 174(5)485-489.

11. Milenović A, Knežević P, Vučićević Boras V, Gabrić D, Andabak Rogulj A, et al. (2015) Influence of neck dissection on the corresponding motor and sensory nerves 137(7): 219-223.

12. De Jong AA, Manni JJ (1991) Phrenic nerve paralysis following neck dissection. Eur Arch Otorhinolaryngol 248(3): 132-134.

13. Spiro RH, Strong EW, Shah JP (1994) Classification of neck dissection: Variations on a new theme. Am J Surg 168(5): 415-418.

14. Yaddanpudi S, Shah SC (1996) Bilateral phrenic nerve injury after neck dissection: an uncommon cause of respiratory failure. J Laryngol Otol 110(3): 281-283.

15. Sayama J, Shineha R, Hirayama K, Nishihira T, Mori S (1994) Four cases of phrenic nerve paralysis following surgery of thoracic esophageal carcimona. Nihon Kyobu Geka Gakkai Zasshi 42(4): 562-567.

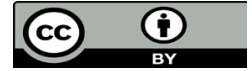

This work is licensed under Creative Commons Attribution 4.0 License

To Submit Your Article Click Here:

Submit Article
DOI: 10.32474/SJO.2018.01.000102

\section{Scholarly Journal of Otolaryngology}

\section{Assets of Publishing with us}

- Global archiving of articles

- Immediate, unrestricted online access

- Rigorous Peer Review Process

- Authors Retain Copyrights

- Unique DOI for all articles 